\title{
CORPS ET SEXUALITÉ CHEZ LES ROMANCIÈRES TUNISIENNES
}

Enjeux de reconnaissance, coûts et effets des «transgressions » Abir Kréfa

\section{La Découverte | Travail, genre et sociétés}

\author{
$2011 / 2-n^{\circ} 26$ \\ pages 105 à 128
}

ISSN 1294-6303

Article disponible en ligne à l'adresse:

http://www.cairn.info/revue-travail-genre-et-societes-2011-2-page-105.htm

Pour citer cet article :

Kréfa Abir, «Corps et sexualité chez les romancières tunisiennes » Enjeux de reconnaissance, coûts et effets des «transgressions »,

Travail, genre et sociétés, 2011/2 n²6, p. 105-128. DOI : 10.3917/tgs.026.0105

Distribution électronique Cairn.info pour La Découverte.

(c) La Découverte. Tous droits réservés pour tous pays.

La reproduction ou représentation de cet article, notamment par photocopie, n'est autorisée que dans les limites des conditions générales d'utilisation du site ou, le cas échéant, des conditions générales de la licence souscrite par votre établissement. Toute autre reproduction ou représentation, en tout ou partie, sous quelque forme et de quelque manière que ce soit, est interdite sauf accord préalable et écrit de l'éditeur, en dehors des cas prévus par la législation en vigueur en France. II est précisé que son stockage dans une base de données est également interdit. 


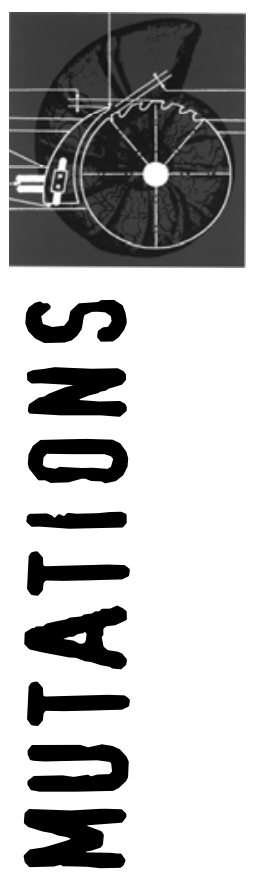

CORPS ET SEXUALITÉ

CHEZ LES ROMANCIÈRES TUNISIENNES

Abir Kréfa

LES FILLES DANS LES ÉCOLES

SUPÉRIEURES DE COMMERCE EN FRANCE

PENDANT L'ENTRE-DEUX-GUERRES

Marianne Thivend

FEMMES ET RICHESSE :

AU-DELÀ DU PIB

Florence Jany-Catrice et Dominique Méda 


\title{
CORPS ET SEXUALITÉ CHEZ LES ROMANCIÈRES TUNISIENNES
}

\section{ENJEUX DE RECONNAISSANCE, COÛTS ET EFFETS DES «TRANSGRESSIONS »}

\footnotetext{
I 1 n'est aujourd'hui plus possible de reprendre, comme le font certain-e-s critiques de la littérature dite «maghrébine» [Segarra, 1997, p.7], la célèbre phrase de Kateb Yacine en préface au roman de Yamina Mechakra: «une femme qui écrit vaut son pesant de poudre» [1986, p. 8]. Fondée sur un présupposé culturaliste et essentialisant, cette réitération méconnait les mutations réelles qu'ont connues au cours des quarante dernières années chacun des espaces littéraires des pays du «Maghreb». S'agissant du cas qui nous intéresse ici, celui de la Tunisie, les femmes y ont commencé par publier, d'abord sous pseudonyme, dans les années 1950 [Fontaine, 1990], en même temps que commençait à émerger un espace éditorial où l'imprimé a supplanté le manuscrit [Bendana, 2001]. Dans les années 1960, elles n'hésitent plus à signer des œuvres qui se caractérisent par une très forte tonalité de dénonciation des structures religieuses et patriarcales. C'est le cas, en 1968, de Leila Ben Maami avec son recueil de nouvelles Sawma'a tahtariq (Minaret en flamme) et, en 1979, de Hayet Bencheikh avec son recueil Bila Rajoul (Sans homme). En 1975, sont publiés de façon concomitante les trois premiers romans
}

\author{
Abir Kréfa* \\ * Je remercie Isabelle \\ Charpentier, Amélie \\ Le Renard et Guillaume \\ Malochet pour leur \\ relecture et leurs \\ remarques sur des \\ versions antérieures à \\ ce texte.
}


en langue française écrits par des femmes et recensés par la critique : Cendres à l'aube de Jalila Hafsia [1975], La Vie simple de Souad Guellouz [1998] et Rached d'Aïcha Chabbi [1975]. Les femmes demeurent toutefois largement minoritaires pour accéder à l'édition. Ainsi, sur les 183 romans de langue arabe publiés entre 1983 et 2001, seulement dix-sept l'ont été par des femmes [Fontaine, 2001].

Les analyses féministes des œuvres écrites par des femmes s'attachent souvent à examiner si la réappropriation de la représentation d'elles-mêmes peut contribuer à déstabiliser les rapports de pouvoir en produisant un ordre symbolique éventuellement alternatif ou concurrent à l'ordre patriarcal dominant. Dans la mesure où le corps et la sexualité des femmes sont au cœur des édifices symboliques inégalitaires [Bourdieu, 1992], ces lectures s'intéressent au premier chef aux représentations véhiculées à leur sujet [Détrez et Simon, 2006]. En prenant pour objet le corps et la sexualité dans des œuvres romanesques féminines de la Tunisie contemporaine, ce n'est pas cet angle d'approche que nous avons choisi d'adopter, lequel n'apparaît pas ici approprié. En effet, les approches féministes ordinairement mobilisées pour analyser les textes écrits par des femmes partent du présupposé que les productions artistiques sont socialisatrices [Lacoste-Dujardin, 2008], à savoir que les représentations des rapports entre les sexes qu'elles véhiculent peuvent avoir des effets - qui restent à déterminer - sur les croyances et les pratiques de leurs usagere-s [Lahire, 2001]. Or, quand bien même il serait possible de montrer que ces représentations infléchissent les croyances et les pratiques dans le sens d'un plus grand égalitarisme, leurs effets ne peuvent être que limités en raison de l'étroitesse du public de la production littéraire tunisienne. Contrairement à la plupart des pays européens, où il est possible de distinguer deux types de littératures, l'une à production large, destinée à une commercialisation à grande échelle et qui joue souvent le

${ }^{1}$ La question de la féminisation ne se pose pas de la même manière en arabe, des termes féminins existant, antérieurement à tout usage féministe, en particulier mou'allifa (auteure), kâtiba (écrivaine), adîba (écrivaine de littérature) et mouthaqafa (intellectuelle). rôle d'une littérature alimentaire pour ses auteur-e-s ${ }^{1}$, la seconde à production restreinte destinée essentiellement aux pairs [Bourdieu, 1992], l'examen de la structure de l'espace littéraire tunisien ne permet pas de distinguer ces deux pôles. Les écrivain-e-s interviewé-e-s font partie des auteur-e-s contemporain-e-s les plus légitimes et les plus visibles et, pourtant, le nombre de tirage avoisine en moyenne 1500 exemplaires et rares sont les romans qui connaissent plus d'une réédition. Dans ce contexte, le public se limite pratiquement à celui des pairs. S'il n'est pas possible de transposer mécaniquement les analyses de Pierre Bourdieu [1992] à notre objet, on peut toutefois faire apparaître deux pôles autour desquels se structure l'espace littéraire tunisien contemporain. Deux

108 A Travail, genre et sociétés n²6 - Novembre 2011 
critères principaux d'évaluation des œuvres sont fréquemment mobilisés par les trois instances de légitimation (les journalistes, les universitaires et les pairs) qui permettent de distinguer un pôle où ne sont mis en avant que des critères proprement esthétiques et narratifs et un autre qui assigne à la littérature le rôle d'enfreindre des interdits sociaux et/ou politiques. Un retour sur les acceptions données au terme d'adab permet de resituer ces oppositions dans le temps long. Comme le rappelle Richard Jacquemond, jusqu'à la fin du XIX ${ }^{\mathrm{e}}$ siècle, $a d a b$ a désigné tout à la fois « une qualité morale (le savoir-vivre, la politesse), et un corpus écrit (un ensemble d'œuvres)» [2007, p. 364]. Les luttes pour la définition de la «modernité » littéraire, entendue comme la dissociation des deux acceptions de l'adab, ont pour enjeu la construction d'un espace autonome doté de critères d'évaluation qui lui sont propres. Pour les agents littéraires qui se situent à l'opposé du pôle proprement esthétique, la "transgression » des tabous tenus pour structurels dans la société tunisienne contemporaine - à savoir la sexualité, la religion et la politique constitue un des critères d'identification d'une "bonne » œuvre. Une attention particulière est accordée aux « transgressions » sexuelles, à savoir à la mise en scène de personnages dont les pratiques sexuelles, contrevenant aux normes sociales dominantes, ne se situent pas dans le cadre exclusif du mariage. Or, ce critère d'évaluation des œuvres défavorise les femmes qui, à l'inverse des hommes, apparaissent confrontées à une contradiction entre ces normes internes à l'un des pôles de l'espace littéraire, d'une part, et les normes sociales externes, d'autre part. En effet, le plus fort contrôle social exercé sur leur sexualité, ajouté à une lecture fréquemment biographique de leurs œuvres, les expose à une censure sociale disséminée. Irréductible à la seule censure administrative et étatique, cette dernière est fréquemment exercée sur les écrivaines par des membres de l'entourage familial et conjugal. L'approche choisie ici consiste donc à resituer les œuvres dans la diversité des contextes sociaux de leur production, où les normes ne sont pas nécessairement univoques, celles qui sont internes à l'espace littéraire pouvant entrer en contradiction avec les normes externes. Pour cela, il faut rompre non seulement avec les approches féministes qui s'en tiennent à la seule analyse interne des œuvres, mais aussi avec les présupposés de la sociologie de la littérature qui a largement fondé ses analyses sur l'idée d'un écrivain asexué, mais de fait masculin, et qui s'est par conséquent désintéressée des 
${ }^{2}$ C'est ainsi à partir de la théorie de « l'art pour l'art » et de la figure de Flaubert que Pierre Bourdieu [1992] a élaboré sa théorie du champ littéraire, en reprenant le présupposé largement dominant chez les écrivain-e-s et les critiques littéraires de la neutralité des critères de littérarité du point de vue du genre. Pour une analyse intégrant la catégorie de genre, voir [Naudier, 2000].

${ }^{3}$ Sur la difficile construction d'un espace-temps littéraire chez les romancières algériennes contemporaines, voir Christine Détrez [2008]. asymétries de genre endogènes aux espaces littéraires ${ }^{2}$. En prenant l'exemple du corps et de la sexualité, il s'agit de montrer que les critères de légitimité littéraire ne sont pas neutres. Ils produisent des effets différenciés selon la place de l'auteur-e dans les rapports sociaux de sexe. On se propose ainsi de fournir une explication à la sous-représentation des femmes dans l'espace littéraire tunisien qui ne se réduit pas à la mobilisation de causes externes, même si les difficultés à concilier leurs carrières littéraires avec les exigences professionnelles et familiales ${ }^{3}$ jouent incontestablement un rôle important.

L'objectif de cet article est également de montrer en quoi le genre façonne la production littéraire. Loin de verser dans un essentialisme qui postulerait l'existence de deux littératures, l'une «féminine » et l'autre "masculine», dont on chercherait des caractéristiques reliées à des différences naturelles irréductibles, il s'agit au contraire de mettre en évidence comment les romancières tunisiennes ont tenté de résoudre la contradiction entre les normes des pairs et les normes sociales externes. $\mathrm{Si}$ elles ont développé des stratégies narratives, elles puisent également dans d'autres ressources. Par conséquent, on montrera d'abord la place accordée par les pairs aux thématiques sexuelles, les «transgressions » servant à mesurer le degré d'autonomie et de «modernité » des œuvres. On verra ensuite comment cette injonction défavorise les femmes en raison de la censure sociale disséminée qu'elles doivent affronter. Enfin, on décrira les stratégies et les diverses ressources qu'elles mobilisent afin de résoudre la contradiction entre les normes des pairs et la censure à laquelle elles sont confrontées.

\section{Sources}

Cet article repose sur un travail de terrain effectué dans le cadre d'une thèse de doctorat en sociologie, sur les écrivain-e-s tunisien-ne-s contemporain-e-s. Afin d'éviter tout substantialisme, j'ai inclus des écrivains parmi les interviewé-e-s. Le matériau empirique est constitué par des entretiens ainsi que par des sources écrites. Trente-neuf entretiens semi-directifs ont été menés, essentiellement en Tunisie, entre 2006 et 2009, auprès d'écrivain-e-s de littérature, romancier-e-s et/ou nouvellistes, de langue arabe et/ou française. Tou-te-s disposent d'une certaine visibilité et reconnaissance actuellement en Tunisie. Vingt-sept ont été lauréat-e-s du prix «Comar», institué en Tunisie depuis 1997. Les femmes étant faiblement représentées parmi ces dernier-e-s (elles sont au nombre de neuf), j'ai été conduite à effectuer six entretiens supplémentaires auprès d'écrivaines qui sont aussi relativement reconnues au plan national, soit qu'elles aient publié dans des maisons d'édition légitimes (en Tunisie, en Égypte et au Liban), soit qu'elles aient été lauréates d'autres prix (du ministère de la Culture, de la francophonie, etc.). Ces entretiens ont porté

110 A Travail, genre et sociétés n 26 - Novembre 2011 
sur les trajectoires sociobiographiques des écrivain-e-s, leurs carrières littéraires ainsi que sur la censure éventuellement exercée par leur entourage familial et conjugal. Ils ont, la plupart du temps, été effectués dans la langue d'écriture des interviewé-e-s. Les sources écrites sont constituées de textes critiques consacrés à la « littérature féminine » (al adab ennissấ $\hat{\imath}$ ) ainsi que d'un corpus de romans écrits par des femmes. Toutefois, j'ai parfois anonymisé les citations des enquêté-e-s, notamment lorsqu'elles portent sur les conditions privées d'exercice de la création.

\section{NORMES DES PAIRS ET ENJEUX SYMBOLIQUES DES « TRANSGRESSIONS »}

«Entremetteuse Tunis, avec son Avenue vaste à contenir nombrils à l'air et chevelures voilées, laissés-pour-compte, intellectuels, piétons, insolents, fonctionnaires, amants anachroniques, corps qui se frôlent ou se cognent. Confusion des sens et des désirs. Désirs que la narratrice cultive sur fond de rue pour celui qu'elle finira à la fois par aduler et braver. Dans le paysage littéraire tunisien, une voix qui s'élève avec audace ${ }^{4}$ ».

La quatrième de couverture de ce roman de Kaouthar Khlifi [2008], écrivaine de langue française, constitue une bonne illustration des critères de "modernité » (al hadâtha) littéraire valorisés aujourd'hui par les éditeurs/trices tunisien-ne-s. Une grande proportion des écrivain-e-s, hommes et femmes rencontré-e-s, quelle que soit leur langue d'écriture, expliquent le relatif succès de leurs ouvrages, ainsi que ceux de leurs pairs, par leur caractère audacieux. "Audace » $(a l$-jor' $a)$ et «modernité » sont définies à l'aune de la transgression des trois «tabous ${ }^{5}$ qu'écrivain-e-s et éditeurs/trices tiennent pour structurels dans la société tunisienne contemporaine: la politique, la religion et la sexualité. Cette définition de la modernité littéraire résulte de la conjonction de plusieurs phénomènes. Elle tient d'abord à la place dominée des espaces littéraires arabes relativement aux littératures européennes [Casanova, 2008], particulièrement forte s'agissant du roman, genre largement lié à l'histoire (post) coloniale. C'est ainsi dès la fin du XIX siècle, environ quatre décennies après l'émergence en France de la théorie de «l'art pour l'art » et des revendications de l'autonomie de l'artiste par rapport aux normes sociales dominantes [Bourdieu, 1992], que les écrivain-e-s arabes ont placé au cœur de leurs œuvres les thématiques sexuelles [Jacquemond, 2003].

Toutefois, ils/elles ont endogénéisé cette «modernité » en puisant dans le patrimoine érotique de la littérature arabe classique. L'autonomie est défendue relativement aux ulémas ${ }^{6}$, dont il s'est agi de contester la sacralisation de la langue arabe, langue du Coran, mais aussi relativement à des "masses" supposées plus conservatrices et religieuses. Ces logiques de distinction [Bourdieu, 1979] sont très nettes chez les écrivain-e-s

\footnotetext{
${ }^{4} C^{\prime}$ est moi qui souligne.
}

${ }^{5}$ Les écrivain-e-s ont, au cours des entretiens, utilisé tantôt le terme de «tabouhât » (tabous), tantôt celui de «mouharramât » (interdits religieux).

\footnotetext{
${ }^{6}$ Savants religieux chargés d'interpréter le Coran et les textes de la tradition prophétique.
} 
interviewé-e-s, dont la plupart se déclarent largement « désislamisé-e-s » ou « athé-e-s ». Cette clôture de la «modernité » en littérature, en termes d'audace des thématiques, a été favorisée dans le contexte tunisien par la répression politique des intellectuel-le-s islamistes [Khiari, 2003; Hibou, 2006]. Cette dernière a éradiqué toute velléité de formation d'une élite attachée aux valeurs considérées comme islamiques. Elle s'explique enfin par les luttes de classement qui ont opposé, dans la Tunisie post-coloniale, écrivain-e-s de langue arabe et écrivain-e-s de langue française pour imposer la langue littéraire légitime. Répondant aux critiques des francophones sur l'incapacité de la langue du Coran de tout dire, les écrivain-e-s arabophones ont redoublé les «transgressions » et leur production n'a aujourd'hui, de ce point de vue, plus rien à envier à celle de leurs pairs francophones. L'analyse de la place du corps et de la sexualité dans les œuvres romanesques ne permet ainsi pas de relever des différences significatives selon la langue d'écriture, au sens où les écrivaines de langue française seraient plus «transgressives » ou « subversives ». Selon certain-e-s spécialistes de la « littérature maghrébine» francophone, l'écriture en français permettrait aux femmes de s'affranchir des tabous sociaux, et notamment de dire le corps et la

${ }^{7} C^{\prime}$ est ce que soutient Ana Isabel Labra [2005], qui s'appuie sur les œuvres des romancières algériennes Assia Djebar et Malika Mokeddem.

\footnotetext{
${ }^{8}$ Les écrivains sont très souvent nés de parents analphabètes, de mères au foyer et de pères paysans ou ouvriers.
} sexualité. Ces travaux reprennent souvent à leur compte les affirmations de quelques écrivaines de langue française qui doivent plutôt être replacées dans les conflits internes aux espaces littéraires et portant sur la définition de la langue d'écriture légitime. Certes, les femmes sont moins sous-représentées parmi les écrivain-e-s de langue française. Ainsi, en Tunisie, sur les trente-sept romans de langue arabe distingués par le prix Comar entre 1997 et 2009, six ont été publiés par des femmes, alors que c'est le cas de quatorze des quarante romans de langue française.

Toutefois, on ne saurait voir dans ce phénomène un choix linguistique stratégique par les écrivaines, dans la mesure où la majorité des romancières francophones, scolarisées dans des établissements français (catholiques ou laïcs), ne maîtrisent pas l'usage écrit de la langue arabe. Ce phénomène s'explique par des facteurs étrangers à la langue d'écriture, qui tendent à restreindre le recrutement des écrivaines à la bourgeoisie culturelle et/ou économique, laquelle constitue la fraction la plus francisée de la population. Près des trois quarts des écrivains masculins sont originaires des milieux sociaux les plus démunis, tant en capital économique qu'en capital culturel $^{8}$, alors que $c^{\prime}$ est le cas de seulement un cinquième des écrivaines. C'est que, jusqu'à la fin des années 1970, l'accès à l'enseignement supérieur - qui constitue souvent une des

$112 \Delta$ Travail, genre et sociétés n² 26 - Novembre 2011 
conditions non-dites d'entrée dans le champ littéraire - était limité, pour les femmes, à celles issues de la bourgeoisie. Par ailleurs, la possibilité pour des femmes de s'investir dans des pratiques d'écriture qui sont à la fois chronophages et faiblement rémunératrices, est conditionnée par la détention d'un capital économique important, qui rend possible une externalisation des travaux domestiques et/ou une libération de la profession dominante. L'impossibilité de vivre de leur plume contraint, en effet, la majorité des écrivain-e-s à exercer une profession rémunératrice, le plus souvent dans l'enseignement (secondaire ou supérieur) ou le journalisme.

Plus que la langue d'écriture, ce sont les différences générationnelles qui expliquent les variations d'une écrivaine à une autre. Ainsi, les œuvres de la romancière de langue arabe Massouda Aboubaker - née une vingtaine d'années plus tard que la francophone Souad Guellouz - mettent en scène, contrairement aux romans de celle-ci, des personnages féminins aux pratiques hédonistes. C'est le cas, en particulier de Rîm, dans Wadâ'an Hamourâbi (Adieu Hamourâbi) [Aboubaker, 2003], qui décide de faire la découverte de la sexualité au sein d'une relation fondée très largement sur l'attirance physique. $\mathrm{Et}$, alors que son amant songe à inscrire leur relation dans la durée, elle lui oppose l'immédiateté du désir et la jouissance temporaire. Dans les cinq lettres qu'elle lui envoie d'Irak, c'est l'expression du désir qui domine et non la déchirure sentimentale provoquée par l'éloignement de l'être aimé, les conceptions de l'amour et de la sexualité que l'auteure attribue aux deux personnages prenant ici le contre-pied de leur distribution sociale habituelle entre les sexes.

Repousser les frontières du dicible en matière de corps et de sexualité est donc devenu un moyen de constituer ou d'accroître son capital symbolique pour un-e auteur-e. Ainsi, dans l'ouvrage qu'il consacre aux œuvres des romancières tunisiennes contemporaines, Bouchoucha Ben Jom'a [2009] loue « l'audace » de celles qui mettent en scène des personnages aux pratiques sexuelles "transgressives", qu'il s'agisse de la prostitution, de l'homosexualité, ou de la sexualité prémaritale. L'évocation du sexe, indépendamment de toute orientation féministe, fait désormais partie de l'horizon d'attente des critiques et des pairs, si bien que celles et ceux qui ne s'y conforment pas se voient reprocher par ces dernièr-e-s un certain "puritanisme». En raison de la faiblesse des publications critiques dans un espace littéraire émergent, ces prescriptions ont souvent lieu de façon informelle, dans les cercles d'interconnaissance. Ainsi, Amel Nkhili a pu nous narrer comment, suite à la publication de son premier roman [2006], des critiques et des pairs masculins lui ont fait remarquer que 
${ }^{9}$ On peut ainsi requalifier ce que Richard Jacquemond [2003, p. 56] appelle une « loi des libertés décroissantes » dans l'Égypte contemporaine - plus le public des biens culturels est large, plus le degré de censure étatique est élevé - qui est également valable pour la Tunisie. celui-ci comporte le défaut de manquer « d'audace » en matière de sexe. Définis dans la perspective de rompre avec les normes sociales dominantes, ces critères de "modernité » n'ont pu s'imposer qu'en raison de l'absence d'une littérature commerciale à destination d'un large public. En effet, la relative autonomie dont bénéficient les écrivain-e-s s'explique, dans le contexte de la censure d'État, par une loi des «libertés croissantes » qui autorise des marges d'autant plus importantes que le bien culturel est faiblement diffusé. Les pièces de théâtre de Jalîla Baccar et Fâdhel J'aỉbi, lorsqu'elles sont diffusées à la télévision, sont ainsi censurées des passages jugés contraires aux «bonnes mœurs ». C'est par exemple le cas du spectacle Familia, pour lequel le visa de représentation a été facilement obtenu pour qu'il soit joué en salle mais qui, lors de sa transmission à la télévision, a été censuré du passage où l'actrice principale demande successivement à trois hommes de l'embrasser dans le cou.

Mais l'élaboration des critères de "modernité » littéraire n'est pas neutre du point de vue du genre. Elle a été effectuée par un milieu de la critique (universitaire comme journalistique) très largement masculin, si bien que les femmes écrivains se sont vues imposer le critère des «transgressions sexuelles » comme étalon de mesure de la "modernité » d'un texte, sans véritablement participer à sa construction. S'il existe une forte interconnexion entre le milieu des écrivains et celui de la critique, ce sont très majoritairement des hommes qui occupent une double position, en tant que producteurs et juges de la littérarité des œuvres. Alors que près des trois quarts des écrivains interviewés, exerçant les professions de journalistes ou d'universitaires, ont publié des textes critiques parallèlement à leurs œuvres de création, c'est le cas de seulement un dixième des écrivaines. Très souvent enseignantes dans le secondaire, la majorité des écrivaines apparaissent en effet, de part leur profession dominante, éloignées des milieux de la critique. Définis par les hommes, les critères de «modernité » littéraire semblent par ailleurs fonctionner au désavantage relatif des écrivaines.

\section{"TRANSGRESSIONS »: UN COÛT PLUS ÉLEVÉ POUR LES ÉCRIVAINES}

\section{Une censure sociale disséminée}

Officiellement, la censure n'existe pas, puisque l'article $1 \mathrm{du}$ Code de la presse assure « la liberté de la presse, de publication, d'impression, de distribution et de vente de livres et publications ». Par ailleurs, l'article 8 de la Constitution tunisienne déclare que « les libertés d'opinion, d'expression, de la presse,

$114 \Delta$ Travail, genre et sociétés n 26 - Novembre 2011 
de publication sont garanties et exercées dans les conditions définies par la loi ». Toutefois, la loi, justement, contraint l'éditeur/trice ou l'écrivain-e ainsi que l'imprimeur-e à l'obligation dite $\mathrm{du}$ « dépôt légal ». Celle-ci consiste à informer le ministère de l'Intérieur, de la Culture, ainsi que la Bibliothèque nationale, de la prochaine mise en circulation de l'ouvrage sur le marché, les administrations devant en retour fournir un récépissé de « dépôt légal ». En réalité, cette obligation est interprétée dans la pratique, par les agent-e-s de l'espace littéraire, ainsi que par les fonctionnaires administratif-ve-s, selon que le récépissé est octroyé ou non, comme l'autorisation ou l'interdiction de la commercialisation de l'ouvrage. Dans les faits, il existe donc une censure préalable. Au moins deux écrivaines, parmi les plus visibles dans leur espace littéraire national, ont au cours des dernières années fait les frais de la censure étatique. Le roman de Fadhîla Châbbi, El 'Adl (La Justice) [2008], fut interdit à la commercialisation pendant quatre ans. Il est possible ${ }^{10}$ que l'histoire du livre, celle d'un général signant, par erreur, l'ordre de castrer toute l'armée, soit à l'origine de sa censure, bien que le lieu du récit ne soit pas spécifié. L'ouvrage d'Amel Mokhtar [2002], El korssi el hazzâz (Le fauteuil à bascule), dont la sortie a été programmée par les éditions Cérès pour l'année 2002, n'a pu être mis sur le marché qu'en 2008. Probablement jugé « pornographique » par le censeur, on peut également supposer que la description des viols subis par la narratrice au cours de son enfance a été le motif de son interdiction.

Cependant, il est ici important de ne pas se limiter à la seule acception étatique et administrative de la censure, et de prendre en considération ses formes davantage disséminées, en particulier lorsqu'elle est exercée par des membres de l'entourage familial ou conjugal. Peu abordée publiquement par les écrivain-e-s et non politisée, la censure se manifeste de façon plus invisible. Elle est autrement plus efficace que la censure étatique, qui peut paradoxalement avoir pour effet - quoique seulement pour celles et ceux qui sont relativement bien insérée-s dans les réseaux de sociabilité littéraires ou journalistiques de publiciser l'ouvrage censuré et, par là, d'accroître le capital symbolique de l'auteur-e. En effet, la censure d'État suscite des formes de résistance collective, comme le montre la tentative de créer une Ligue des Écrivain-e-s Libres ${ }^{11}$, pour protester contre l'immixtion du politique dans le littéraire. Mais les contraintes ne s'exercent pas uniquement en amont de la publication. À cette conception élargie de la censure, il faut ajouter une conception analogue de la réception, qui n'est pas uniquement guidée par un parti pris anti-légitimiste [Grignon et Passeron, 1989]. En privilégiant l'étude de la réception par les instances chargées d'évaluer les œuvres, les analyses classiques de la sociologie de la littérature ne sont pas transposables à notre

\author{
${ }^{10}$ Les motifs de la \\ censure préalable \\ n'étant pas \\ systématiquement \\ notifiés, il ne nous est \\ ici possible que de \\ formuler des \\ hypothèses.
}

${ }^{11}$ C'est en juillet 2001 que sept écrivains (tous masculins), sympathisants des oppositions démocratiques, et contestant la légitimité de la très officielle Union des écrivain-e-s tunisiens à représenter les intérêts des écrivain-e-s, en ont notifié la demande. Malgré les refus de la préfecture de Tunis, puis du ministère de l'Intérieur, d'en autoriser la création, cette initiative aura été l'occasion d'une prise de parole- certes brèvedans certains journaux, afin de publiciser la censure d'État. 
${ }^{12}$ Ce phénomène est également observable pour les écrivaines algériennes. Voir pour cela Christine Détrez [2008].

\footnotetext{
${ }^{13}$ Si les écrivains peuvent aussi faire relire le manuscrit à leur épouse avant sa publication, leurs motifs ne relèvent pas du même ordre. Il s'agit pour eux de tester la bonne transmission des émotions ou du " message " aux lecteurs/trices.
}

objet car les écrivaines peuvent être attentives autant à la réception de leurs œuvres au sein de leur sphère privée que dans la presse ou par les spécialistes de la critique littéraire.

À l'inverse de la censure d'État, ces contraintes s'exercent spécifiquement sur les écrivaines. Les entretiens montrent que la censure des proches, ainsi que les craintes d'une réception réprobatrice de leurs ouvrages par ces derniers, sont fortement ressenties. Totalement ignorées par les écrivains masculins rencontrés, elles ont été expérimentées par six des écrivaines. Il apparaît également que ce sont toujours un ou plusieurs hommes qui font figure de censeurs, institués en gardiens du patronyme $^{12}$. Une romancière décrit ainsi la réaction de son époux lorsqu'elle l'a informé qu'elle allait entamer une carrière littéraire :

«Mon mari avait peur que je me lance dans des écrits qui n'étaient pas conformes à une certaine éthique, qui auraient dévoilé certaines intimités de la famille, il avait peur que je me lâche, que j'écrive pour un public un peu sale. On m'a toujours dit "mets un peu d'érotisme et de porno dans tes bouquins et ça marchera mieux", je n'ai jamais voulu le faire ».

Il n'est toutefois pas nécessaire que les réticences ou les lignes à ne pas franchir soient clairement exprimées aux écrivaines. Même en leur absence, celles-ci peuvent ressentir des contraintes fortes, comme l'explique cette écrivaine, qui a publié son premier roman et qui était connue du monde des lettres avant son mariage :

«Heureusement que j'ai publié mon premier roman avant mon mariage, parce que ce n'est pas évident, par la suite, que tu continues à écrire, tout en étant mariée, à avoir la responsabilité du foyer, et à avoir surtout quelqu'un qui comme ça... essaie de voir ce que tu mijotes.

Enquêtrice : parce qu'il vous censurait?

Non, il ne me censurait pas, mais c'est gênant quand même, parce qu'avant la publication, bon, c'est une liberté absolue, c'est ton truc, donc personne n'a le droit de voir ce qui se passe, le jour où tu décides de le publier, il devient public. Mais avant ça, moi, j'avais horreur que quelqu'un lise mes brouillons par exemple et donc le fait que bon, il y a toujours quelqu'un pas très loin, c'est gênant ».

Par ailleurs, les écrivaines peuvent prendre elles-mêmes l'initiative de soumettre le manuscrit à l'un de leurs proches afin de s'assurer que son contenu n'est pas dérangeant pour leur entourage $\mathrm{e}^{13}$, comme le montre le témoignage de cette romancière :

«Mon mari l'a lu avant que je le publie, parce que comme c'était quand même assez audacieux, ce n'était pas pour lui demander la permission, mais il fallait qu'il soit d'accord, sur certaines choses, ce n'est pas autobiographique du tout, mais enfin quand je dis pas du tout, ce n'est jamais vrai, il y a toujours une part d'autobiographie dans un roman et donc

116 A Travail, genre et sociétés n²6 - Novembre 2011 
bon, il l'a lu, [...] il a aimé, il m’a encouragée à le publier, il était content que

je le fasse ».

En aval de la publication, seules des écrivaines ont eu à affronter une réception réprobatrice de leur entourage et/ou de leur public. C'est ainsi le cas d'une romancière qui, confrontée à l'hostilité d'un époux opposé à ses premières publications dans les journaux, prend l'initiative de divorcer. Si son second époux est plus respectueux de sa carrière littéraire, elle a dû faire face à l'un de ses frères qui lui « reprochait» de décrire, dans ses romans, les scènes amoureuses et sexuelles de ses personnages. Elle a également, sur son lieu de travail, dû affronter la réception moraliste de l'un de ses lecteurs masculins, venu lui signifier qu'elle pouvait faire l'économie de ces scènes.

\section{Réduction autobiographique et inégalités de genre dans la sphère des pratiques sexuelles}

La censure disséminée à laquelle sont exposées les femmes dès qu'elles traitent $\mathrm{du}$ corps et de la sexualité tient à la conjonction de deux phénomènes. D'une part, leurs écrits, beaucoup plus que ceux des hommes, et quand bien même ils adopteraient le genre fictionnel, font l'objet d'une réduction autobiographique, à savoir une interprétation biographique qui leur dénie leurs capacités d'imaginer et de " fabuler » ${ }^{14}$ et donc, finalement, de créer. À quoi il faut ajouter l'asymétrie réelle des normes de genre dans la sphère des pratiques sexuelles. La censure sur les questions de sexualité, qui ne s'exerce qu'à l'encontre des femmes et uniquement par des hommes, s'explique par le surinvestissement du corps des femmes en tant que dépositaire de l'honneur du groupe. Dans un système patriarcal, c'est en effet, comme le rappelle Sophie Ferchiou [1989], « la vertu des femmes qui constitue le support essentiel de l'honneur du groupe [...] et ce sont les hommes [...] qui sont investis de l'autorité nécessaire pour contrôler cette sexualité dangereuse » (pp.83-84). Interprétées comme le résultat de transgressions réellement franchies, celles qui sont opérées dans leurs écrits suscitent, beaucoup plus que pour les hommes écrivains, l'opprobre de leur entourage. Car si, depuis les analyses de Sophie Bessis et Souhayr Belhassen [1992], on peut observer une relative évolution des rapports de genre dans la sphère des pratiques sexuelles et un desserrement du contrôle sur la sexualité des femmes - notamment préconjugale - dans certaines fractions de la bourgeoisie intellectuelle, cela ne signifie pas que soit toléré le dévoilement public de telles pratiques.

\footnotetext{
${ }^{14}$ Selon l'expression d'Aroussia Nalouti, dans un entretien mené avec elle.
} 
Certaines écrivaines ont expérimenté très tôt la réduction autobiographique de leur œuvre par leur entourage, dès leurs publications de jeunesse. Elles ont alors dû faire œuvre de critique littéraire et dire le hiatus séparant la fiction et la vie, en insistant sur la spécificité de la littérature. Ainsi de cette romancière dont la parution de la première nouvelle, pourtant très en deçà de «l'audace » aujourd'hui prônée par les pairs, a suscité des réactions vives de son père, petit commerçant ayant eu une scolarité courte. Bien que finalement convaincu du caractère fictionnel du récit, celui-ci lui signifie qu'elle doit s'imposer des limites, manière de lui faire comprendre qu'il s'institue en gardien du patronyme :

"Je me rappelle, cette nouvelle, ce qu'elle a pu me coûter comme... Quelqu'un est venu voir mon père pour lui dire "ah c'est ta fille qui a écrit je sais pas quoi, elle parle de quelqu'un qui l'a enchaînée et je ne sais pas quoi", et là il est venu me dire : "tu veux donc me déshonorer !". Je lui ai dit "mais c'est rien, c'est imaginaire", il m'a dit "imaginaire? Moi je sais que c'est vrai !", donc ce soupçon d'autobiographie remonte à ce moment-là, c'est-à-dire que ce que tu écris ne peut être qu'autobiographique. Après, c'est comme si mon père a eu honte, il est venu me dire "mais je sais que tout ce qui s'écrit n'est pas réel! Bon, mais, mais il ne faut pas écrire n'importe quoi !" ".

Toutefois, ce ne sont pas seulement les agents éloignés des milieux littéraires qui procèdent volontiers à la confusion entre l'ordre de la fiction et celui du vécu, entre l'auteure et son (ou ses) personnage(s), mais également les critiques littéraires euxmêmes. Tout en concédant que l'écriture du moi n'est pas spécifique aux écrivaines, Bouchoucha Ben Jom'a la considère comme le trait autorisant à parler d'une « littérature féminine ». Si la mention fréquente du terme « roman » sur la page de couverture est destinée à attester du caractère fictionnel du récit, il interprète les œuvres romanesques féminines comme des autobiographies déguisées, les auteures ne pouvant selon lui révéler des pratiques sexuelles transgressives au grand jour: «dans leurs textes romanesques, l'ensemble des écrivaines se couvrent pudiquement d'un voile à l'aide de noms d'emprunts qu'elles attribuent à leurs hérö̈nes, dans le but d'annuler la possibilité de les tenir pour des autobiographies » [2009, p. 20]. L'universitaire et romancier Mahmoud Tarchouna, autre spécialiste reconnu de la «littérature féminine», commence par pointer les problèmes que pose une interprétation autobiographique, à la fois parce que les écrivaines ne détiennent pas le monopole de l'écriture autobiographique et parce que les données autorisant une telle interprétation font souvent défaut [2003, p. 14]. Cette précaution méthodologique ne l'empêche cependant pas de se livrer à cet exercice et le retrait de certaines

$118 \Delta$ Travail, genre et sociétés n 26 - Novembre 2011 
écrivaines de la scène littéraire, après une ou deux publications, est expliqué par leur incapacité à puiser ailleurs que dans leur expérience individuelle pour produire la trame d'un récit [2003, pp. 71-72].

Or, la réduction biographique à laquelle procède souvent la critique est fortement ressentie par les écrivaines, comme le montre ce témoignage :

"C'est toujours un homme qui critique une femme, malheureusement nous n'avons pas de critiques femmes, et je pense que c'est très important qu'on en ait [...] ces hommes-là arrivent avec quand même leur idéologie [...] Il y a une déformation qui existe chez beaucoup de lecteurs hommes, et de critiques, que si une femme parle d'aventures amoureuses, ils vont dire "celle-là les a vécues", ils ne le disent jamais à propos des hommes ! [...] et parfois c'est un peu mesquin, c'est un peu naï à la limite ».

Il n'est, par conséquent, nullement étonnant que les femmes déclarent beaucoup plus souvent s'autocensurer que les hommes. Elles entretiennent un rapport réflexif à la censure, qui n'est donc pas uniquement intériorisée et inconsciente, comme le montre le témoignage de cette romancière :

«Enquêtrice: Vous vous autocensurez quand vous écrivez ou pas, sur certains... sur certaines choses?

Certainement, certainement, je crois qu'il y a des non-dits, et à chaque fois je rêve de les chatouiller un peu, au moins pour pouvoir les sortir, bon ils sortent un tout petit peu mais ce n'est pas tout à fait ça, il y a des choses que je n'oserai pas peut-être, ils sont tout le temps enveloppés par un style qui les suggère mais qui ne les montre pas ».

La réduction autobiographique a plusieurs conséquences. La critique masculine prend la forme d'un voyeurisme, où on cherche l'auteure derrière les personnages, supposés être des doubles de la première [Planté, 1989]. D'autre part, si, comme l'estiment les romancières elles-mêmes, les productions littéraires ne sont pas sans lien avec leurs expériences vécues, la réduction autobiographique a pour effet de leur dénier les facultés imaginatives et réflexives, et donc créatrices. Enfin, elle soumet les écrivaines à des injonctions contradictoires - celles des pairs et des normes sociales externes à l'espace littéraire ainsi qu'à l'ambivalence des critiques.

\section{LES STRATÉGIES EXTRA-NARRATIVES}

Comment, dès lors, les écrivaines composent-elles avec ces contraintes ? Autrement dit, comment parviennent-elles tout de même à éditer et quelles stratégies ont-elles mobilisées afin de résoudre la contradiction entre les normes internes et les normes externes à l'espace littéraire ? 


${ }^{15}$ Sur l'usage du
pseudonyme dans
d'autres contextes,
respectivement chez les
romancières
algériennes
contemporaines et les
écrivaines européennes
du XIXe siècle, voir
Christine Détrez [2008,
pp. 85-86] et Christine
Planté [1989, pp. 32-34].

${ }^{15}$ Sur l'usage du pseudonyme dans respectivement chez les romancières algériennes Christine Détrez Planté [1989, pp. 32-34]

\section{Pseudonymes et patronymes}

Contrairement aux hommes, les femmes doivent souvent se poser la question du nom à apposer sur leurs œuvres. Afin d'éviter à leur entourage la déconsidération symbolique induite par leurs écrits, elles peuvent publier sous pseudonyme ${ }^{15}$. Or cette solution de repli, qui les nie comme individu-e-s créatrices, n'est pas toujours garante d'une plus grande marge de liberté. Car, pour parvenir à se faire éditer, la mobilisation d'un réseau relationnel est souvent nécessaire, les démarches directes auprès des éditeurs/trices aboutissant rarement. Par ailleurs, souvent originaires de la bourgeoisie, les écrivaines sont aisément identifiées par les éditeur-trice-s, qui peuvent prendre la décision de censurer certains passages de leurs écrits. Bien qu'issues de milieux sociaux plus privilégiés que leurs homologues masculins, les écrivaines ne bénéficient pas ici d'un privilège de classe qui viendrait compenser leur assignation au mauvais genre social. C'est ainsi le cas de Hafidha Karabibène Yimmen, nouvelliste, poétesse et romancière de langue arabe, investie dans les cercles de sociabilité littéraire tunisois dans les années 1970, qui fait le choix de publier son premier recueil de nouvelles sous le pseudonyme de Bent $A l$ Bahr (Fille de la mer). Ayant rencontré l'éditeur en mobilisant son capital social, celui-ci censure l'une de ses nouvelles au motif des déconsidérations symboliques qu'elle porterait à son père - qui bénéficiait d'un certain prestige à la fois en tant que notable religieux et poète $-s$ 'instituant ainsi lui-même en gardien du patronyme. Les tentatives de l'auteure de convaincre l'éditeur que son père lui-même ne trouverait rien à redire à la nouvelle auront été vaines et le recueil a été donc amputé. Lorsque les écrivaines sont en couple, et qu'elles repoussent l'éventualité du pseudonyme, elles ont le choix de publier soit sous leur patronyme de naissance, soit sous celui de leur mariage, soit les deux. Les règles sociales sont ici relativement souples pour les femmes. Comme pour les interactions ordinaires, ainsi que pour leur identité administrative, elles les autorisent à faire le choix de l'un ou de l'autre, ou des deux. L'une des interviewées, craignant les retombées négatives de ses écrits pour son mari et ses enfants, fait le choix de publier sous son patronyme de naissance, plus répandu en Tunisie que celui de son mari. Toutefois, les écrivaines peuvent également refuser leur relative invisibilisation et faire au contraire un usage stratégique du patronyme, en misant sur celui-ci. C'est ainsi le cas d'Alia Mabrouk, issue de la bourgeoisie provinciale tunisienne et de la petite bourgeoisie française, mariée à 22 ans à celui qui deviendra un grand banquier tunisois. L'organisation des réceptions mondaines qui lui incombe lui a permis de se faire un «nom» dans la bourgeoisie tunisoise, où elle

120 A Travail, genre et sociétés $n^{\circ} 26$ - Novembre 2011 
compte ses principaux lecteurs et lectrices. Malgré les réticences initiales de son mari et de sa belle-famille, elle publie sous son nom marital :

"Quand j'ai commencé à écrire, tout le monde a poussé des hauts cris en me disant "mais tu es folle qu'est-ce-qui te prends ?" J'ai pris le nom de mon mari en me disant qu'après tout, Béji, c'est le nom de mon père, Mabrouk c'est le nom de mon mari et en fin de compte le seul que j'ai dans la société actuellement, c'est Alia Mabrouk, c'est le nom que je me suis fait moi [...]. Donc la famille de mon mari a grincé des dents, en disant "attention, c'est notre nom", donc ça n'a pas été très bien accueilli au départ, même par mon mari ».

Enfin, les écrivaines peuvent, lorsque ce sont uniquement les réactions de la famille élargie qui sont craintes, trouver un moyen terme entre la reconnaissance envers un père qui a joué un rôle important dans leur socialisation littéraire et à qui elles sont liées par une relation affective forte ${ }^{16} \mathrm{~d}^{\prime}$ une part, et la préservation symbolique de son patronyme d'autre part. En effet, les femmes se recrutent plus souvent que les hommes dans des milieux bien dotés en capital culture ${ }^{17}$, la plupart du temps détenu par un homme ${ }^{18}$. Alors qu'aucun des hommes n'a eu dans son entourage familial de personne liée aux métiers du livre ou exerçant des activités para-littéraires, c'est le cas de cinq des femmes rencontrées. Deux sont nées d'un père écrivain, une d'un père et d'une tante écrivain-e-s, et deux ont eu un grand-père imprimeur ou libraire. Au travers des discussions littéraires avec leurs filles, de la mise à leur disposition d'ouvrages de littérature, de leurs encouragements à écrire et à publier, ces pères que les écrivaines présentent comme «modernistes » ou "progressistes » ont eu un rôle important dans leur carrière littéraire. D'origine provinciale, née d'un père qui l'a beaucoup incitée à effectuer des études supérieures, à écrire et publier, l'une des romancières signe ainsi - de ses premiers écrits dans la presse et dans les revues littéraires jusqu'à ses œuvres romanesques - par son prénom accolé à celui de son père. À ces "identités stratégiques" [Collovald, 1988] et à ces variations dans la présentation de soi s'ajoute la mobilisation d'autres ressources.

\section{Des ressources diverses}

La comparaison des trajectoires matrimoniales fait ressortir des différences significatives selon le sexe des enquêté-e-s. Alors que tous les hommes, sauf un, sont installés de façon continue en couple depuis leur mariage, la moitié des femmes sont actuellement célibataires ${ }^{19}$. Parmi celles-ci, deux ne se sont jamais mariées, quatre sont divorcées, tandis que deux sont veuves depuis une dizaine d'années. Les écrivaines peuvent d'abord bien sûr, face à un époux censeur, prendre l'initiative

\footnotetext{
${ }^{16}$ Sur l'importance de ce facteur dans la transmission de l'héritage, en particulier culturel, voir Bernard Lahire [1995, pp. 30-31].

${ }^{17}$ Delphine Naudier [2007] a mis en évidence des résultats similaires pour les lauréat-e-s du Goncourt.

${ }^{18}$ En effet, nées dans les années 1920-1940, les mères des écrivaines ont eu faiblement accès à la scolarisation. Si trois sur quinze ont été scolarisées, elles sont diplômées du secondaire, alors que six écrivaines ont un père diplômé d'études supérieures.

${ }^{19}$ Les taux de célibat ont connu une nette augmentation au cours de la dernière décennie, passant de $17,7 \%$ à $37,5 \%$, entre 2001 et 2006, pour les femmes âgées entre 30 et 34 ans [Enquête nationale sur la population et l'emploi, 2008]. Toutefois, les romancières interviewées sont nées entre les années 1930 et 1960 et constituent des cas atypiques par rapport à l'ensemble des femmes de leurs générations.
} 
d'un divorce, parfois en bénéficiant de l'appui d'un membre de leur entourage et, très souvent, elles font alors le choix de ne pas se remarier. C'est le cas d'une des écrivaines rencontrées, née dans les années 1950. Elle s'est mariée à 33 ans, après avoir repoussé plusieurs projets de mariage, par peur de compromettre sa carrière littéraire. Pendant les quatre années de sa vie conjugale, elle a fait l'expérience de la censure, par l'intermédiaire de son ex-époux, lui-même romancier, qui ne bénéficiait pas de la même notoriété qu'elle :

«Il me censurait, il voulait être au courant de ce que j'écrivais ou pas, il se mêlait de beaucoup de choses, il m'interdisait même de publier. On était deux personnes de culture mais moi j'étais au sommet de ma gloire et de ma renommée, j'avais une grande présence et j'étais très connue, et lui était un écrivain respecté, mais il n'avait pas cette grande présence. Quand il $m^{\prime}$ interdisait de faire quelque chose, je ne la faisais pas, je restais figée. Après j'ai commencé à perdre mon souffle petit à petit (littéralement, " j'ai commencé à mourir », bdét nmout), et je suis entrée dans une phase de grand désespoir, jusqu'à ce que ma famille découvre mon visage jaune, ma terreur. Ma mère m'a encouragée à divorcer et c'est comme ça que le divorce a eu lieu ».

Afin de déjouer les réductions biographiques et signifier la distance qui les sépare de leurs personnages, les écrivaines peuvent faire un usage stratégique de leur statut matrimonial, au cours de leurs rencontres publiques avec les journalistes ou les critiques. C'est ce qu'explique l'auteure d'un roman dont l'héroïne est une jeune femme célibataire qui entretient un rapport hédoniste à la sexualité :

«Pendant les rencontres dans les espaces culturels, au moment des débats et des réceptions, il y avait pratiquement tout le temps mon mari et ma mère avec moi, et quelquefois mes enfants aussi, et alors les hommes journalistes étaient étonnés, ils étaient un peu déçus parce que quand tu lis, tu te dis "merde ça doit être quelqu'un de complètement défoncé, tant qu'à faire on va en profiter", et alors j'arrive avec le truc très clean, traditionnel, emmerdant, je dis "voilà, je vous présente mon mari, ma mère, mes enfants" (elle rit). "Ah bon, vous êtes mariée ?" Des fois ça m’amusait, donc je faisais exprès d'emmener surtout mon mari ».

Lorsqu'elles sont célibataires, les écrivaines doivent mobiliser d'autres ressources. Dans la mesure où elles sont de grandes lectrices, elles peuvent mettre en avant leur capital culturel pour dissocier l'œuvre et la vie. Face au problème que pose à son frère la parution d'un article au sujet de l'un de ses romans, cette romancière, âgée d'une quarantaine d'années, qui ne s'est jamais installée en couple, met ainsi en évidence la différence entre connaissance pratique et connaissance livresque :

« Je me rappelle d'un article qui a causé un problème entre mon frère et moi, il était écrit que j'avais une grande connaissance de la psychologie, du sexe et tout ça, et donc pour mon frère, sa sœur, qui est connaisseur... ! Je lui ai 
dit, écoute, on n'est pas obligé de connaître quelque chose par la pratique, vous me voyez tout le temps en train de lire, je lis souvent les revues et les

livres sur la psychologie, je lis aussi des livres sur le sexe, sur tout ».

Les écrivaines sont donc contraintes d'insister, dans leurs interventions publiques comme dans leurs discussions avec leur entourage, sur la nécessaire dissociation de l'œuvre et de la vie, et de dire la distance qui sépare leurs pratiques sociales de celles de leurs personnages. Ce faisant, elles sont loin de se situer sur le plan de la délégitimation des inégalités de genre dans la sphère des pratiques sexuelles réelles. Elles cherchent en effet d'abord à être reconnues en tant qu'écrivaines.

\section{STRATÉGIES ET EFFETS LITTÉRAIRES}

\section{Dissoudre le récit}

L'un des procédés fréquemment utilisés par les romancières pour déjouer les réductions biographiques consiste en la clôture de l'œuvre littéraire sur elle-même. La narratrice, ou le principal personnage féminin, est ainsi souvent elle-même écrivaine, et l'histoire du livre apparaît comme celui de sa propre genèse, où le récit est dissous. Cette technique du roman dans le roman et de l'enchâssement est utilisée par au moins deux des écrivaines actuellement reconnues dans l'espace littéraire tunisien. Tamâss [Nalouti, 1995] alterne ainsi des épisodes de la vie de Zeynab Hassan et de Zeynab 'Abdeljabbâr, la seconde étant le personnage du roman de la première. Certes, la technique du roman dans le roman est loin d'être spécifique aux écrivaines dans la mesure où on la retrouve également chez les écrivains masculins. Le Paradis des femmes ainsi que Hayet ou la passion d'Elles, romans respectifs d'Ali Bécheur [2006] et d'Anouar Attia [2002] narrent la genèse du roman lui-même à travers une ou plusieurs figures féminines allégoriques, où l'amour du narrateur pour celles-ci et pour la littérature sont confondus. Toutefois, les significations que les un-e-s et les autres accordent à ce procédé apparaissent fortement genrées. Chez les écrivains masculins, le leitmotiv de l'amour et du désir comme prétextes au texte est directement lié aux modes d'entrée dans l'écriture. En effet, les lettres d'amour rédigées à l'adolescence ont joué un rôle important dans leur incorporation des dispositions littéraires. Chez les écrivaines, il s'agit de stratégies narratives destinées à décourager toute réception biographique. Les écrivaines entretiennent un rapport réflexif à ces usages, qu'elles relient explicitement aux contraintes auxquelles elles sont confrontées, comme le montre l'interview d'Aroussia Nalouti.

«Les critiques sont toujours en train d'attendre, à la fin de la séance officiel-

le, pour venir vous dire : "vraiment j'ai aimé cet épisode, parce qu'il y a..." 
tout ça pour vous demander "vous ne l'avez pas vécu par hasard ?", donc voilà, ici l'écrivaine écrit une histoire, son personnage lui-même écrit une autre histoire, de telle manière qu'on se demande mais où sont les limites entre la fiction et la réalité, c'est pour dérouter le lecteur donc, lui dire tu ne sauras pas trouver où je me situe ».

Le brouillage des frontières entre la réalité et l'imaginaire est également l'un des effets de la structure narrative du roman d'Amel Mokhtar El korssi El hazzâz [2002]. Si elle a connu un accueil public favorable à son premier roman, édité dans la très légitime maison beyrouthine Dâr al adâb [Mokhtar, 1992], elle a été en revanche parallèlement accusée, au sein des cercles d'interconnaissance littéraires et journalistiques, "d'outrage aux mœurs » et soupçonnée de mener une vie aussi «dissolue » que sa narratrice qui, en outre, parle à la première personne. Elle complexifie donc la structure narrative dans son second roman. Mouna, le principal personnage féminin, qui est aussi la narratrice, cherche à regagner l'affection de son père. Celui-ci a subi un choc terrible en la voyant faire l'amour avec l'un de ses amants sur son fauteuil attitré, symbole du patriarche. Mouna prend alors la décision d'accepter la proposition de mariage d'un collègue, dont elle pense qu'il est assez aisé pour être un bon parti aux yeux de son père. Cependant, le soir de ses noces, elle s'enfuit avec l'un des amis de son époux, et passe une semaine avec lui en Espagne. Ce sont les diverses versions de la perte de sa virginité qui forment la suite du roman. À chacun de ses interlocuteurs masculins - amants, père et amis -, elle tient un récit différent, si bien qu'elle finit par douter d'une quelconque vérité des faits. La narratrice étant elle-même écrivaine, plus on avance dans le roman, plus se renforce l'hypothèse que les « faits » sont en partie fictionnels. Le brouillage des frontières entre la réalité et la fiction est renforcé par la dédicace du roman à Hâmed 'Abd Essalâm, le père, non de l'auteure, mais de la narratrice. À travers ces procédés qui interrogent les frontières entre le réel et l'imaginaire, c'est toute tentative de réduction biographique de l'œuvre qui est découragée.

\section{Multiplier les personnages}

Les écrivaines ont également souvent recours à la multiplication des personnages féminins. Dans Chronique frontalière, Emna Bel Haj Yahia [1991] met en scène deux personnages, Zeïneb et Narjess, opposés en plusieurs points. La première est mariée, mère de deux enfants et enseignante. « À douze ans, le harem était déjà dans sa tête » [p.10]. Toutefois, elle est écartelée entre le respect des traditions et l'insoumission: " jamais il n'y eut pareil mélange d'acceptation et de refus » [p. 20]. Elle est tout au long du roman tiraillée entre les deux 
cultures qu'elle a reçues au cours de son enfance: l'une religieuse («ce grand Olivier par les doigts de sa mère en elle planté » [p. 46]), l'autre sécularisée, celle de ses livres d'écolière. Quant à Narjess, orpheline de père et mère très jeune, elle incarne la révolte contre les normes patriarcales, en affichant volontiers un anticonformisme vestimentaire ou en décidant de transgresser la norme de la virginité en se trouvant un amant à 17 ans. Si elle se marie ensuite, c'est «par goût intermittent du risque » [p. 78] et met rapidement un terme à son mariage. Elle incarne ce que Zeïneb ne peut réaliser elle-même. Au travers de ces deux personnages, sont mises en récit les illusions et désillusions des femmes tunisiennes nées autour de l'Indépendance : la croyance dans le discours de libération des femmes et la prise de conscience que la société tunisienne demeure structurée par de profondes inégalités entre les sexes. Par là, le roman d'Emna Bel Haj Yahia se situe dans la lignée de l'approfondissement du projet réaliste $\mathrm{du}$ roman arabe. Sa bonne réception dans l'espace littéraire tunisien s'explique par les bénéfices symboliques conférés par une publication dans une maison d'édition parisienne, mais aussi par sa conformité à certaines des normes littéraires valorisées.

Bien qu'écrit en langue arabe, 'Adhrâ khârje al mizân, le roman de Fâtma Cherîf [1999], présente de nombreuses similitudes avec Chronique frontalière. Autoédité, il a toutefois été vendu à plus de 20000 exemplaires et a permis à son auteure d'accéder à une certaine reconnaissance dans le centre de la littérature arabe, au Caire ${ }^{20}$. Il narre la relation à leur corps de deux personnages, Aïcha et Saïda, toutes deux âgées entre 25 et 30 ans. Aïcha tombe amoureuse d'un homme qui tente de la convaincre de coucher avec lui, en stigmatisant l'archaïsme de la virginité prémaritale. Bien qu'attirée, Aïcha s'y refuse, parce qu'elle craint d'être ensuite méprisée et rejetée par lui. Elle découvre qu'il la trahit avec son amie Saïda et qu'il a également formulé à celle-ci la promesse de l'épouser prochainement. À la fin du roman, toutes deux apprennent qu'il part enseigner dans le Golfe et que son mariage avec sa cousine est imminent. À travers les propres tiraillements d'Aïcha, la relation contrastée à leurs corps qu'ont les deux amies, et les inconséquences du personnage masculin, ce roman réaliste et psychologique met en lumière les injonctions contradictoires auxquelles sont soumises les femmes arabes des grandes villes. Le personnage masculin se sera moqué des deux femmes, de celle $q^{\prime} u^{\prime} i l$ traite $\mathrm{d}^{\prime}$ « arriérée » et de celle qui apparaît « libérée». Le roman ne délivre aucun message normatif: ni valorisation de la virginité, ni non plus apologie d'une liberté

\author{
${ }^{20}$ Sur le rôle du Caire \\ et de Beyrouth dans \\ l'accès à la \\ reconnaissance \\ panarabe des \\ écrivain-e-s arabes, \\ voir Richard \\ Jacquemond [2003] et \\ Franck Mermier [2005].
}


sexuelle, qui dans le cadre des rapports de genre existants, n'apparaît pas possible.

Louees et encouragées par les pairs, les «transgressions 》 sexuelles sont au cœur de la définition de la «modernité » des œuvres parmi la génération d'écrivain-e-s dominant aujourd'hui l'espace littéraire tunisien. Alors que ces «transgressions » peuvent accroître le capital symbolique d'un-e auteur-e, elles comportent un coût social diffus pour les femmes, particulièrement fort dans la sphère privée. L'analyse de la contradiction entre les normes externes et celles des pairs fournit ainsi une explication à leur éviction de l'espace littéraire. Celle-ci ne constitue toutefois qu'une des modalités de leur exclusion. En même temps que les femmes ont commencé à investir l'espace littéraire, à partir des années 1960, une catégorie ségrégative - «la littérature féminine » - a émergé, qui particularise les œuvres des femmes relativement à la littérature masculine tenue pour référent universel, et les nie comme individus créateurs. C'est la convergence des intérêts de trois acteurs qui a conduit à l'institutionnalisation de cette catégorie et à son incorporation dans les pratiques : les critiques littéraires, l'État tunisien, ainsi que les éditeurs. Enfin, si l'on replace les écrivaines tunisiennes dans «La République mondiale des lettres » [Casanova, 2008], l'examen des conditions de la réception de leurs œuvres dans l'espace éditorial et littéraire français montre les difficultés pour elles d'accéder à une reconnaissance à la fois nationale et internationale. Alors que la dénonciation de la domination masculine en terre arabomusulmane constitue l'une des conditions pour être éditée ou traduite en France, les critiques littéraires ainsi que les éditeurs/trices tunisien-ne-s tendent à associer une valeur négative à cette thématique. Les écrivaines qui ont été reconnues par la critique littéraire tunisienne (Aroussia Nalouti, Nefla Dhab) ont été justement celles qui ont pris leurs distances avec la dénonciation des structures patriarcales qui dominait dans les premières œuvres féminines. Grâce à leur capital culturel élevé, elles se sont davantage investies dans le travail narratif et esthétique. Or ces écrivaines, comme toutes celles qui se situent dans le pôle esthétique de l'espace littéraire tunisien, n'ont guère de chance d'accéder à une reconnaissance littéraire en France, qui constitue l'un des centres littéraires dominants au niveau international. 


\section{BIBLIOGRAPHIE}

ABOUbAKER Massouda, 2003, Wadâ'an Hamourâbi (Adieu Hamourabi), Tunis, Éditions Cérès.

ATTIA Anouar, 2002, Hayet ou la passion d'Elles, Tunis, Éditions Cérès. BECHEUR Ali, 2006, Le Paradis des femmes, Tunis, Élyzad.

BENCHEIKH Hayet, 1979, Bila rajoul (Sans homme), Tunis, autoédition.

Bel Haj YAHIA Emna, 1991, Chronique frontalière, Paris, Noël Blandin.

BEN MAAMI Leila, 1968, Sawma'a tahtariq (Minaret en flamme), Tunis, autoédition.

BENDANA Kmar, 2001, "Générations d'imprimeurs et figures d'éditeurs à Tunis entre 1850 et 1950 ", in MICHON Jacques et MOLLIER Jean-Yves, (dir.), Les Mutations du livre et de l'édition dans le monde du XVIII siècle à l'an 2000, Paris/Québec, L'Harmattan/Presses de l'université Laval, pp. 349-359.

BEN JOM'A Bouchoucha, 2009, Erriwâya ennissâ'iya ettounissiya (Le roman tunisien féminin), Tunis, autoédition.

BESSIS Sophie et BELHASSEN Souhayr, 1992, Femmes du Maghreb: l'enjeu, Paris, Jean-Claude Lattès.

BSAÏS Wafa, 2008, Sais-tu seulement ce que vivre veut dire?, Tunis, Éditions Sahar.

BOURDIEU Pierre, 1979, La Distinction. Critique sociale du jugement, Paris, Éditions de Minuit.

BOURDIEU Pierre, 1998 [1992], Les règles de l'art. Genèse et structure du champ littéraire, Paris, Le Seuil.

BOURDIEU Pierre, 2002 [1998]. La domination masculine, Paris, Le Seuil.

CASANOVA Pascale, 2008 [1999], La République mondiale des lettres, Paris, Le Seuil.

CHABBI Aïcha, 1975, Rached, Tunis, Éditions de la MTE.

CHABвI Fadhîla, 2008, El 'Adl (La Justice), Tunis, autoédition.

CHERIF Fatma, 1999, 'Adhrâ khârej el mizân (Vierge déconcertante), Tunis, autoédition.

Collovald Annie, 1988, «Identité(s) stratégique(s) », Actes de la Recherche en Sciences Sociales, $\mathrm{n}^{\circ} 73$, pp. 29-40.

DETREZ Christine et SIMON Anne, 2006, À leur corps défendant. Les femmes à l'épreuve du nouvel ordre moral, Paris, Le Seuil.

DETREZ Christine, «Domination et résistances à l'épreuve du terrain : le cas des romancières algériennes ", in Marie BUSCATTO, Catherine MARRY et Delphine NAUDIER (dir.), 2008, Travail, genre et art, Document de travail du Mage.

Enquête nationale sur la population et l'emploi, 2008, Tunis, Institut National des Statistiques.

FERCHIOU Sophie, 1989, « Pouvoir, contre-pouvoir et société en mutation : l'exemple tunisien », Peuples méditerranéens, n 48-49, pp. 81-92.

FONTAINE Jean, 1990, Écrivaines tunisiennes, Tunis, Éditions Le Gai Savoir.

FONTAINE Jean, 2001, Le roman tunisien de langue arabe: 1956-2001, Tunis, Éditions Cérès.

GRIGNON Claude et PASSERON Jean-Claude, 1989, Le savant et le populaire, populisme et légitimisme en sociologie et en littérature, Paris, Le Seuil/Gallimard. 
Guellouz Souad, 1998, Myriam ou le rendez-vous de Beyrouth, Tunis, Éditions Sahar.

HAFSIA Jalila, 1975, Cendres à l'aube, Tunis, autoédition.

HiBOu Béatrice, 2006, La force de l'obéissance. Économie politique de la répression en Tunisie, Paris, La Découverte.

JACQUEMOND Richard, 2007, « Un temps de renouvellements littéraires », in Hallaq BOUTROS et Heidi TOELLE (dir.), Histoire de la littérature arabe moderne, Tome I, Arles, Actes Sud, pp. 364-375.

JACQUEMOND Richard, 2003, Entre scribes et écrivains. Le champ littéraire dans l'Égypte contemporaine, Arles, Actes Sud.

JRAD Neila, 1996, Mémoire de l'oubli. Réflexion critique sur les expériences féministes des années quatre-vingt, Tunis, Éditions Cérès.

KHIARI Sadri, 2003, Tunisie, le délitement de la cité. Coercition, consentement, résistance, Paris, Karthala.

KHLIFI Kaouthar, 2008, Ce que Tunis ne m'a pas dit, Tunis, Éditions Élyzad.

LABRA CENITAgoya Ana Isabel, 2005, «Hors du harem linguistique: identité et langues d'écriture chez les romancières maghrébines ", Actas do $i$ simposio internacional sobre o bilingüismo, pp. 211-221.

LACOSTE-DUJARDIN Camille, 2008, La vaillance des femmes. Les relations entre femmes et hommes berbères en Kabylie, Paris, La Découverte.

LAHIRE Bernard, 1995, Tableaux de famille, Paris, Gallimard/Le Seuil.

LAHIRE Bernard, 2001, « Héritages sexués : incorporation des habitus et des croyances", in Thierry BLÖss (dir.), La dialectique des rapports hommesfemmes, Paris, Presses Universitaires de France, pp. 9-25.

MARZOUKI Ilhem, 1993, Le mouvement des femmes en Tunisie au XXe siècle: féminisme et politique, Paris, Maisonneuve \& Larose.

MechaKRa Yamina, (Préf. KATEB Yacine), 1986 [1979], La grotte éclatée, Alger, ENAL.

MERMIER Franck, 2005, Le Livre et la Ville. Beyrouth et l'édition arabe, Arles, Actes Sud.

MOKHTAR Amel, 1992, Nakhb El Hayêt (Le festin de la vie), Beyrouth, Éditions Dâr al adâb.

MOKHTAR Amel, 2002, El Korssi el hazzâz (Le fauteuil à bascule), Tunis, Cérès Éditions.

NAlouti Aroussia, 1995, Tamâss (Tangente), Tunis, Sud Éditions.

NAUDIER Delphine, 2000, La cause littéraire des femmes. Modes d'accès et modalités de consécration des femmes dans le champ littéraire (1970-1998), Thèse de doctorat, Paris, EHESS.

NAUDIER Delphine, 2007, «Les lauréates du Goncourt: une légitimité littéraire en trompe-l'œil ? », in Delphine NAUDIER et Brigitte ROLLET (dir.), Genre et légitimité culturelle. Quelle reconnaissance pour les femmes?, Paris, L'Harmattan, pp. 121-141.

NKHILI Amel, 2006, Tarânîm el bardî el kadîm (Cantiques d'un vieux papyrus), Tunis, Sud Éditions.

Plante Christine, 1989, La petite scur de Balzac. Essai sur la femme auteur, Paris, Le Seuil.

SEGARRA Marta, 1997, Leur pesant de poudre. Romancières francophones du Maghreb, Paris, L'Harmattan.

TARCHOUNA Mahmoud, 2003, Nakd erriwâya ennissâ 'iya fî tounés (Critique du roman féminin en Tunisie), Tunis, Centre de publication universitaire.

$128 \Delta$ Travail, genre et sociétés nº 26 - Novembre 2011 